\title{
Hypoxia-induced miR-191-C/EBP $\beta$ signaling regulates cell proliferation and apoptosis of fibroblast-like synoviocytes from patients with rheumatoid arthritis
}

\author{
Shanshan $\mathrm{Yu}^{1+}$, Ying Lu ${ }^{1,2+}$, Ming Zong ${ }^{1}$, Qi $\operatorname{Tan}^{1}$ and Lieying Fan ${ }^{1 *}$
}

\begin{abstract}
Background: Hypoxia plays an important role in the proliferation of rheumatoid arthritis fibroblast-like synoviocytes (RA-FLS), leading to pathology of RA. This study was conducted to evaluate hypoxia-induced microRNAs (hypoxamiR) in RA-FLS and its role in the function of RA-FLS.

Methods: RA-FLS were cultured under normoxia $\left(21 \% \mathrm{O}_{2}\right)$ or hypoxia $\left(3 \% \mathrm{O}_{2}\right)$ condition, followed by a microRNA (miRNA) array analysis. The upregulation of miR-191 by hypoxia was confirmed in RA-FLS and FLS from osteoarthritis (OA) patients by quantitative real-time polymerase chain reaction (RT-PCR). Transfection of miR-191 mimic and inhibitor was used to investigate the function of miR-191 in RA-FLS. The functional targets of miR-191 were predicted by bioinfomatics and then validated by reporter gene assay.

Results: A subset of miRNAs was identified to be induced by hypoxia including miR-191. The upregulation of miR-191 was found to be specific in hypoxic RA-FLS, compared to hypoxic OA-FLS. We observed that miR-191 in RAFLS increased cellular proliferation via promoting $G_{1} / S$ transition of the cell cycle and suppressed cell apoptosis induced by cell starvation. Bioinformatical analysis and experimental assays identified CCAAT/enhancer binding protein $\beta$ (C/EBP $\beta$ ) as a target gene of miR-191 in RA-FLS. Enforced expression of C/EBP $\beta$ rescued the cellular phenotypes induced by miR-191. In addition, an inverse correlation between the C/EBP $\beta$ level and hypoxia stimulation was found in RA-FLS, and overexpression of C/EBP $\beta$ could partly rescue the hypoxia-induced cell proliferation.

Conclusion: We demonstrated the miR-191-C/EBP $\beta$ signaling pathway mediating the hypoxia-induced cell proliferation in RA.
\end{abstract}

Keywords: miR-191, CCAAT/enhancer binding protein $\beta$, Hypoxia, Rheumatoid arthritis, Fibroblast-like synoviocytes

\section{Background}

Rheumatoid arthritis (RA) is a systemic autoimmune disease, which is characterized by chronic joint inflammation and synovial hyperplasia. It is estimated that there are approximately $1 \%$ of population being affected by this disease worldwide [1]. Rheumatoid arthritis fibroblast-like synoviocytes (FLS), as the main stromal cell population in the joint synovium, proliferate and

\footnotetext{
* Correspondence: flieying@yeah.net

†Shanshan Yu and Ying Lu contributed equally to this work.

${ }^{1}$ Department of Clinical Laboratory, Shanghai East Hospital, Tongji University School of Medicine, $150 \mathrm{Ji}$ Mo Road, Shanghai 200120, People's Republic of China

Full list of author information is available at the end of the article
}

invade to destroy the adjacent cartilage $[2,3]$. The mechanisms regulating the aberrant growth of RA-FLS remain unclear, although quite a few studies have been reported from literature indicating the hypoxia environment in the joint may be the main reason causing inflammation and hyperplasia in RA [4-6].

In comparison with RA, osteoarthritis (OA) is a degenerative condition that is the result of increased wear and tear on joints $[7,8]$. Since the inflammatory symptoms, joint damage and fibroblast-like synoviocytes proliferation are all more severe in RA than that in OA, OA has been frequently used as control to study the pathobiology of RA.

(C) The Author(s). 2019 Open Access This article is distributed under the terms of the Creative Commons Attribution 4.0 International License (http://creativecommons.org/licenses/by/4.0/), which permits unrestricted use, distribution, and 
Reduced oxygen and induced inflammation in the synovium of arthritis have been well demonstrated as the key role in the progression of RA [9]. In the inflamed joint, the level of hypoxia is inversely correlated with the levels of vascularity, oxidative damage, and synovial inflammation [10, 11]. Hypoxia induces angiogenesis and promotes cell growth by regulating the expression of the key genes, such as hypoxia-inducible factors (HIFs) and vascular endothelial growth factor (VEGF) [12]. Our previous study also demonstrated that hypoxia promoted cellular proliferation of RA-FLS and angiogenesis in RA though upregulating glucose-6-phosphate isomerase (G6PI) [13].

In addition, microRNAs (miRNAs) have also been found to express abnormally under hypoxia condition, which is closely related with cell types [14-16]. miRNAs are a class of small non-coding RNA molecules regulating the stability or translational efficiency of targeted messenger RNAs, participating in a wide range of biological processes such as cellular proliferation, differentiation, and apoptosis [17]. miR-155 and miR-146a are the most well-studied miRNAs in RA, significantly upregulated in RA-FLS, and take roles in RA progression and development $[18,19]$. However, the miRNA expression profile and the potential function of hypoxia-regulated miRNAs in RA-FLS have not been systematically studied yet.

In this study, we performed a miRNA screen analysis in the hypoxia-exposed RA-FLS and identified miR-191 induced by hypoxia condition. Overexpression of miR-191 promoted cellular proliferation and protected RA-FLS from apoptosis. CCAAT/enhancer binding protein $\beta(C / E B P \beta)$ was demonstrated to be a target gene of miR-191, mediating the regulatory function of miR-191 in RA-FLS.

\section{Methods}

\section{Specimen collection and HE staining}

Synovial tissues from RA $(n=5)$ and OA $(n=5)$ patients were fixed in $10 \%$ neutral buffered formalin and embedded in paraffin and then cut into 5-um-thick sections, and stained with hematoxylin and eosin (HE), following the manufacturer's introduction. All patients fulfilled the diagnosis of the American College of Rheumatology (ACR) for RA and OA. Informed consent was obtained from each of the enrolled patients, and the study protocol was approved by the Ethics Committee of Shanghai East Hospital (2016-df-011). Prior to tissue collection, signed informed consent was obtained from each patient. This study was conducted in accordance with the guidelines of the Declaration of Helsinki.

\section{Isolation and culture of fibroblast-like synoviocytes}

Synovial tissues from RA or OA patients were immediately placed in RPMI 1640 medium (Life Technologies,
Carlsbad, CA, USA) and processed within $4 \mathrm{~h}$. The tissues were minced and evenly spread on the bottom of cell culture flasks in RPMI 1640 medium at $37^{\circ} \mathrm{C}$ for 6 h. Next, the tissues were incubated with RPMI 1640 medium supplemented with $10 \%$ fetal bovine serum (FBS) and penicillin $(100 \mathrm{U} / \mathrm{ml})$ and streptomycin $(100 \mu \mathrm{g} / \mathrm{ml})$ at $37^{\circ} \mathrm{C}$ in a humidified $5 \% \mathrm{CO}_{2}$ atmosphere. Non-adherent tissue pieces were carefully removed by replacing the medium every 3 to 5 days and passaged when the primary synoviocytes reached 70 $80 \%$ confluence. FLS grown over four to eight passages and were used for further analysis. FLS were cultured under normoxia condition and/or $3 \% \mathrm{O}_{2}$ hypoxia condition in BioSpherix oxygen control system.

\section{RNA quantification and real-time PCR analysis}

Total RNA of FLS was extracted using TRIzol ${ }^{\mathrm{mm}}$ (Invitrogen, Carlsbad, CA, USA), after culture under the conditions of normoxia or $3 \% \mathrm{O}_{2}$ hypoxia for $24 \mathrm{~h}$. An M\&G miRNA Reverse Transcription Kit (miRGenes, Shanghai, China) was used to prepare the first strand cDNA of miRNAs following the manufacturer's instruction. One hundred nanograms of purified total RNA from each sample was used for miRNA measurement. After reverse transcription, the cDNA was diluted 1:1,000 for quantitative real-time polymerase chain reaction (RT-PCR). The miRNA profiling analysis was performed with the quantitative RT-PCR-based miRNA panel which contains 365 miRNAs and 2 reference small RNAs ( $5 \mathrm{~s}$ ribosomal RNA and u6) on an ABI PRISM 7900 (Applied Biosystems, Foster City, CA, USA). Forward primer sequences for RT-PCR of miRNAs were miR-191: $5^{\prime}$ gaatcccaaaagcagctg 3'; miR-297: 5' atgtgtgcatgtgcatg 3'; miR-499b-3p: 5' aacaucacugcaagucu 3'; miR-770: 5' uccaguaccacgugucag 3'; miR-936: 5' acaguagagggaggaaucg 3'. For C/EBP $\beta$ mRNA detection, total RNA was reverse transcribed to complementary DNA (TaKaRa, Dalian, China) according to the manufacturer's instructions. Gene expression was analyzed by relative quantification using Premix Ex Taq SYBR Green PCR (TaKaRa) on an ABI 7500 Real Time PCR System (Applied Biosystems). The sequences of primers were used as follows: $\mathrm{C} / \mathrm{EBP} \beta$, forward: $5^{\prime}$-TTCA AGCAGCTGCCCGAGCC-3', reverse: 5' -GCCAAGTGC CCCAGTGCCAA-3'; and GAPDH, forward 5'- TGAC TTCAACAGCGACACCCA-3', reverse 5' -CACC CTGTTGCTGTAGCCAAA-3'. GAPDH served as the internal control. All RT-PCR reactions were analyzed using Relative Expression Software Tool (REST $\left.{ }^{\circ}\right) 2009$ based on $2^{-\Delta \Delta \mathrm{Ct}}$ method.

\section{Transfection of siRNA and plasmid}

Mimic-miR-191 and its control (control-mimic), and antisense oligo nucleotide targeting miR-191 (inhibitormiR-191) and its control (inhibitor-control) were 
synthesized by ribobio Co. (Guangzhou, China). The target sequences for C/EBP $\beta$ siRNA (si-C/EBP $\beta)-1$ (5' ${ }^{\prime}$ CCG TGG TGT TAT TTA AAG A-3'), si-C/EBP $\beta-2$ (5'-CCC TGA GTA ATC GCT TAA A-3'), and si-negative control (si-NC) (5'-TTC TCC GAA CGT GTC ACG T-3') were synthesized by Genepharm (Shanghai, China). The plasmids of pEnter and pEnter-C/EBP $\beta$ were synthesized by Weizhen (Shandong, china). The transfection of siRNAs and plasmids was performed using Lipofectamine 2000 (Invitrogen, Carlsbad, CA, USA) following the manufacturer's protocol. Final concentration of $30 \mathrm{nM}$ of small RNA oligos was used for all in vitro assays.

\section{Cell proliferation assay}

RA-FLS that had been transiently transfected with small RNA oligos $(30 \mathrm{nM})$ or plasmids $(2 \mu \mathrm{g} / \mathrm{ml})$ for $24 \mathrm{~h}$ were seeded on $4 \times 10^{3}$ cells/well in 96-well plates and incubated under conditions of normoxia or $3 \% \mathrm{O}_{2}$ of hypoxia. At the end of each time period (as indicated in the figures or figure legends) from transfected cells being seeded, cell proliferation was determined by using the Cell Counting Kit-8 (CCK8) (Donjindo, Japan) according to the manufacturer's instructions. Briefly, $20 \mu \mathrm{l}$ CCK 8 was added to each well containing $200 \mu \mathrm{l}$ medium and then incubated at $37^{\circ} \mathrm{C}$ for $2-4 \mathrm{~h}$. The absorbance was read at $450 \mathrm{~nm}$ on a spectrophotometric plate reader (Bio-Rad, Hercules, CA, USA). Each assay was performed in quintuplicate, and all tests were repeated three times.

\section{Cell cycle and cell apoptosis analysis}

RA-FLS were transfected with the indicated oligos or plasmids in six-well plates. For cell cycle analysis, the cells were collected after $48 \mathrm{~h}$ of transfection. The cells were washed with PBS and then fixed with $70 \%$ ice-cold ethanol overnight at $-20^{\circ} \mathrm{C}$, washed with PBS, resuspended with $400 \mu \mathrm{l} \mathrm{PBS}$, and then incubated with $100 \mu \mathrm{g} / \mathrm{ml}$ RNaseA (Kaiji, China) for $30 \mathrm{~min}$ at $37^{\circ} \mathrm{C}$ and with $50 \mu \mathrm{g} / \mathrm{ml}$ propidium iodide (PI) (Kaiji, China) for another $30 \mathrm{~min}$ at $4{ }^{\circ} \mathrm{C}$. After incubation, the cells were subjected to DNA content analysis using BD FACS Calibur cytometry and the results were analyzed with the ModFitLT software. For apoptosis analysis, the complete medium was changed to RPMI 1640 medium supplemented with $1 \%$ FBS to induce cell apoptosis, after transfection for $24 \mathrm{~h}$. Cell apoptosis was evaluated by Annexin V-FITC and PI (BD Biosciences, 556547) staining according to the manufacturer's protocol, followed by flow cytometry analysis. Briefly, cells were collected and washed with ice-cold PBS and resuspended in $100 \mu \mathrm{l}$ binding buffer. Then, $5 \mu \mathrm{l}$ of Annexin V-FITC and $5 \mu \mathrm{l}$ of PI were added to the cells, incubated for $15 \mathrm{~min}$ at room temperature in the dark, and an additional $400 \mu \mathrm{l}$ of binding buffer was added to the reaction prior to analysis. The results were analyzed with the Summit v4.3 software.

\section{Western blot analysis}

Cells were lysed in lysis buffer and centrifuged at 12,000 rpm for $10 \mathrm{~min}$. The protein samples in the supernatant were immediately collected, and the concentration was measured using the Bradford method (Bio-Rad, Hercules, CA, USA). Equal amounts of protein were separated by $10 \%$ SDS-PAGE and then transferred onto PVDF (polyvinylidene fluoride) membranes (Amersham Pharmacia Biotech, Uppsala, Sweden). After blocking with 5\% nonfat dry milk in PBS containing $0.1 \%$ Tween-20 for $2 \mathrm{~h}$ at room temperature, the membranes were incubated at $4{ }^{\circ} \mathrm{C}$ overnight with primary antibodies (1:1000) against cyclinD1 (\#2978, cell signaling), CEBP/ $\beta(\# 3087$, cell signaling), and $\beta$-actin (sc-47778, Santa Cruz) and subsequently incubated with secondary horseradish peroxidase antibody. The immunoreactive proteins were visualized using an enhanced chemiluminescent reagent (Millipore Corporation, USA).

\section{Transwell migration assay}

RA-FLS that had been transiently transfected with inhibitor-control or inhibitor-miR-191 were plated in transwell invasion chambers (Corning) on membranes precoated with Matrigel (Corning) containing RPMI 1640 medium supplemented with 1\% FBS, and RPMI 1640 medium supplemented with 5\% FBS in the lower wells. After a 24-h incubation, Matrigel were removed with a cotton swab, and the cells were fixed and stained with $0.1 \%$ crystal violet solution and assessed by two observers in a blinded manner.

\section{Luciferase reporter assay}

293T cells were seeded on 24-well plates at a density of $1 \times 10^{5}$ cells. Then, cells were transfected with $1.0 \mu \mathrm{g} \mathrm{WT}$ or pMIR-REPORT plasmid (Obio, Shanghai, china) and $100 \mathrm{ng}$ Renilla plasmid, together with mimic-miR-191/ mimic-ctrl $(30 \mathrm{nM})$. After a 24-h transfection, reporter assays were performed using the Dual Luciferase kit (Promega, Madison, WI, USA) by AutoLumat.

\section{Statistical analysis}

SPSS 20.0 program package (SPSS Inc., Chicago, IL, USA) was used for all statistical analyses. Data are presented as mean \pm SEMs. The standard two-tailed Student's $t$ test was used for analysis, in which $p<0.05$ was considered statistically significant.

\section{Results}

miRNA expression screening in hypoxic RA-FLS

The synovial tissue samples from patients with RA $(n=5)$ and OA $(n=5)$ were collected, paraffin-embedded, and 
sectioned, followed by HE staining analysis. As shown in Fig. 1a, RA patients showed inflammation in the lining of the joint and synovial hyperplasia, compared to OA patients. In order to determine the potential role of hypoxia-induced microRNAs (hypoxamiR) in RA-FLS, primary RA-FLS from three patients were isolated and cultured under normal or $3 \% \mathrm{O}_{2}$ of hypoxia for $24 \mathrm{~h}$. Quantitative RT-PCR-based miRNA profiling analysis were performed and compared between normal and hypoxia conditions. Figure $1 \mathrm{~b}$ showed that, out of a total of 365 miRNAs, 5 showed significant upregulation more than 1.5 fold change in all 3 RA-FLS cell lines by hypoxic culture including miR-191, miR-297, miR-499b-3p, miR-770, and miR-936, while only 2 of the 365 miRNAs showed downregulation in 2 of the 3 samples including miR-29b and miR-320d. Furthermore, the expression of the five upregulated miRNAs was compared in three RA-FLS and three OA-FLS cell lines cultured under normal or hypoxia condition. The expression of miR-191 and miR-770 was found to be specifically upregulated in RA-FLS, whereas other three miRNAs (miR-297, miR-499b-3p, and miR-936) showed upregulation by hypoxia treatment in both RA-FLS and OA-FLS (Fig. 1c).

miR-191 promoted the cellular proliferation of RA-FLS

MiR-191 was suggested to be an oncomiR by literature to be aberrantly expressed in various human cancers including breast cancer [20], hepaotocellular carcinoma [21], and colon cancer [22]. In order to elucidate the function of hypoxia-induced miR-191 in cellular proliferation in RA-FLS, mimic or inhibitor oligo of miR-191 was applied. As shown in Fig. 2a, transfection of mimic-miR-191 into RA-FLS increased the level of miR-191 around 100 times, whereas inhibitor-miR-191 decreased the miR-191 levels in RA-FLS efficiently. A CCK8 assay demonstrated the increased proliferation after mimic-miR-191 transfection. On the contrary, miR-191 inhibition decreased cell viability significantly (Fig. 2b). Western blot analysis demonstrated that the protein level of cyclinD1 increased after transfection with mimic-miR-191 (Fig. 2c) and significantly decreased in miR-191-downregulated RA-FLS (Fig. 2d). Cell cycle analysis indicated the promotion of $G_{1} / S$ transition in RA-FLS by miR-191 overexpression (Fig. 2e) and an attenuation of the $G_{1} / S$ transition by miR-191 inhibition (Fig. 2f).

miR-191 inhibited starvation-induced apoptosis in RA-FLS To determine the role of miR-191 in cell apoptosis, the Annexin V/PI analysis was applied to detect the apoptotic cells after transfection of mimic-miR-191 (Fig. 3a), followed by a quantitative analysis (Fig. 3b). Similar assays were performed after transfection of inhibitor-miR-191 (Fig. 3c, d). Quantitative analysis indicated significant decrease in the proportion of apoptotic cells from $6.37 \%$ to $3.45 \%$ by overexpression of miR-191 (Fig. $3 \mathrm{~b}$ ) and increase of apoptotic cells from $8.42 \%$ to 14.2\% by knockdown of miR-191 (Fig. 3d). a

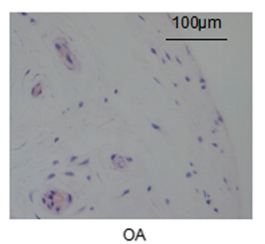

C
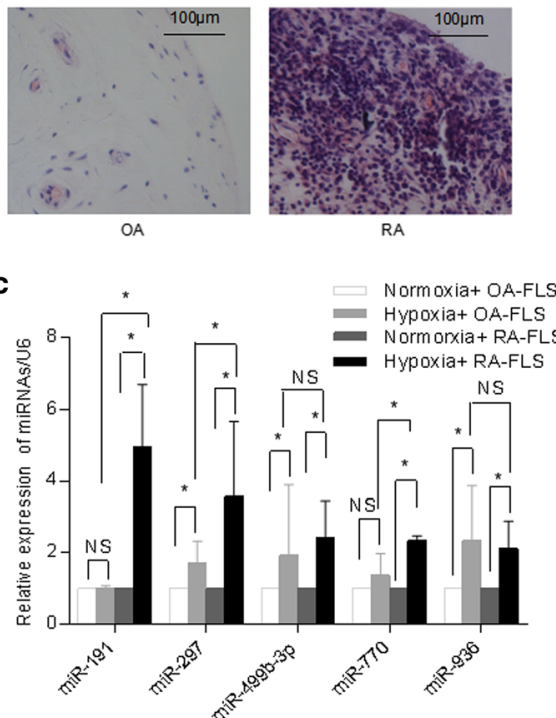

b

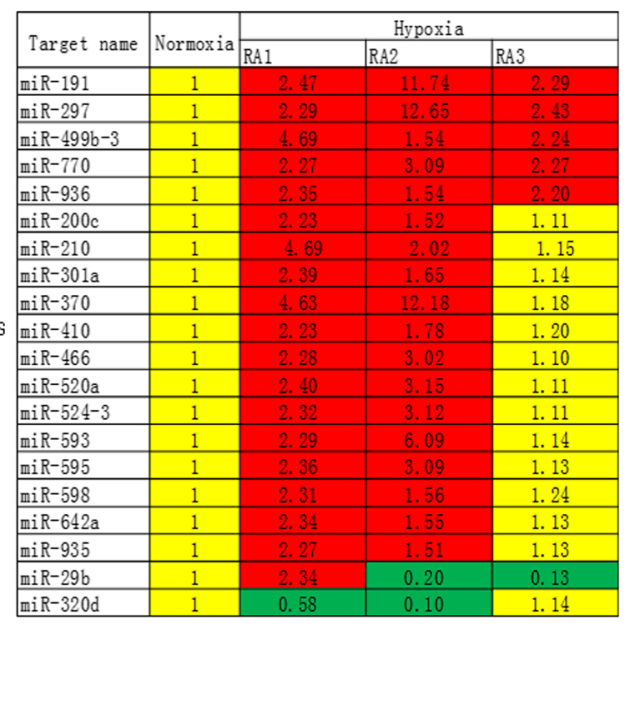

Fig. 1 Upregulation of miR-191 in hypoxic RA-FLS. a HE staining of synovial tissue samples from RA and OA patients. b miRNA profiling analysis for RA-FLS, cultured under normal or hypoxia conditions for $24 \mathrm{~h}$, identified a subset of dysregulated miRNAs. Data was represented as fold change of miRNAs expression in hypoxic RA-FLS, compared to normoxic RA-FLS. c Further validation of the miR-191, miR-297, miR-499b-3p, miR770, and miR-936 expression in three RA-FLS cells and three OA-FLS cultured under hypoxia condition for $24 \mathrm{~h}$, compared to normoxia condition by quantitative RT-PCR. Data represent results from three independent experiments, shown as means \pm SEMs $\left(n=3,{ }^{*} p<0.05\right.$ by $t$ test) 


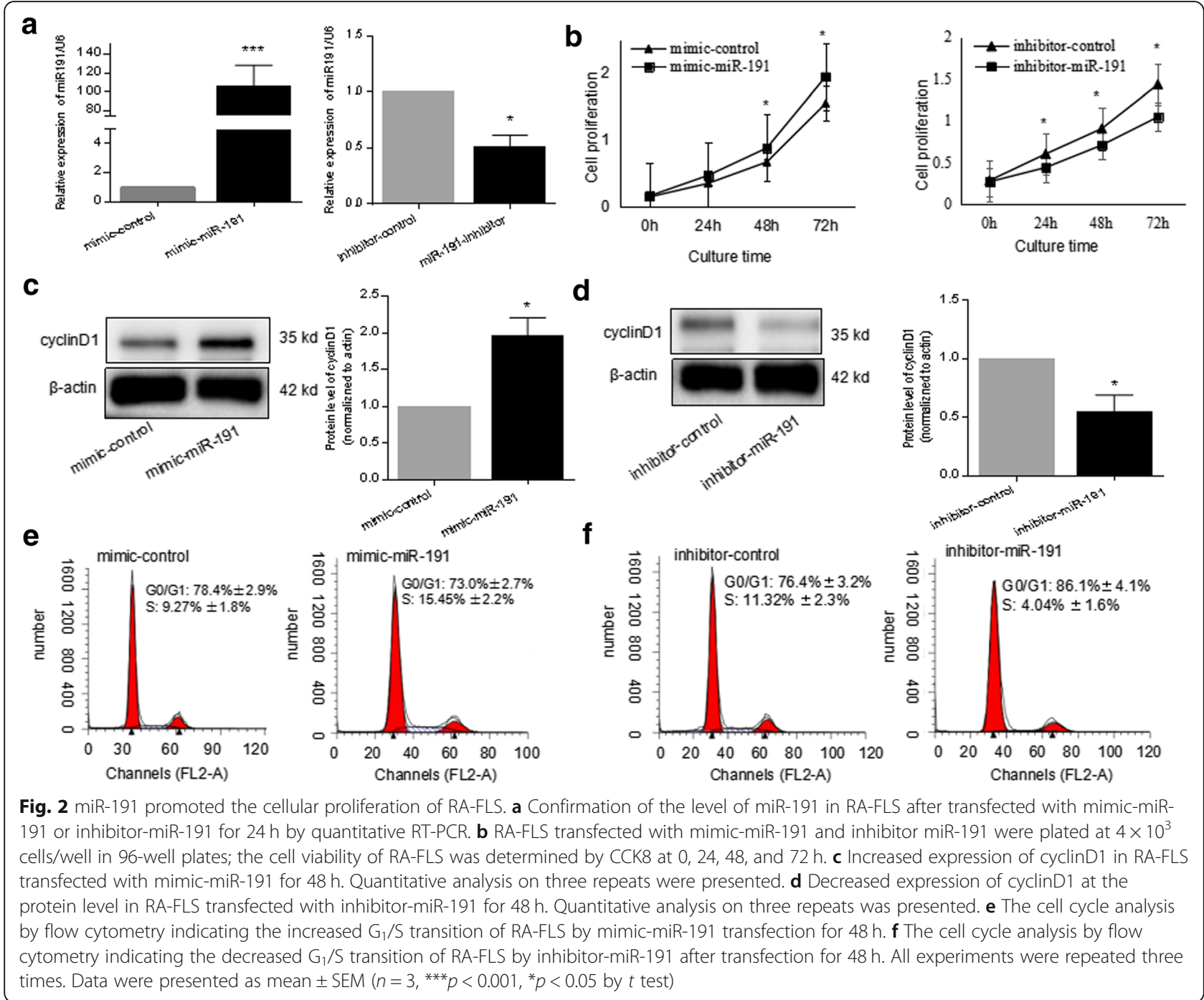

Transwell assays were further applied to determine the cell migration/invasion regulation by miR-191 using RA-FLS cells with or without knockdown of miR-191. As shown in Fig. 3e, knockdown of miR-191 significantly suppressed the cell invasion ability of RA-FLS.

\section{C/EBP $\beta$ is a target gene of miR-191 in RA-FLS}

In order to determine the mechanism through which miR-191 regulate the proliferation of RA-FLS, we used three publicly available databases (TargetScan, picTar, and miRanda) to search for predicted direct target genes of miR-191. We found a binding site of miR-191 in the $3^{\prime}-$ UTR of C/EBP $\beta$ mRNA. C/EBP $\beta$ is a member of the C/EBP family of transcription factors and has been reported to regulate cell proliferation, differentiation, and cell apoptosis in a variety of cells [23-25]. Quantitative RT-PCR and western blot demonstrated that overexpression of miR-191 could remarkably reduce the expression of $\mathrm{C} / \mathrm{EBP} \beta$ at both mRNA level (Fig. 4a) and protein level (Fig. 4b) in RA-FLS. The pMIR-REPORT luciferase vector was established using the wild type 3 '-UTR of C/EBP $\beta$ and a point mutation sequence to the miR-191 binding site (Fig. 4c). As shown in Fig. 4d, miR-191 could directly suppress WT $3^{\prime}$-UTR of C/EBP $\beta$, but not mutant vector.

\section{C/EBP $\beta$ mediated the miR-191 regulation of cell proliferation in RA-FLS}

In order to determine the function of $\mathrm{C} / \mathrm{EBP} \beta$ as the target gene of miR-191 in RA-FLS, siRNAs targeting $\mathrm{C} /$ $\operatorname{EBP} \beta$ (si-C/EBP $\beta-1$ and si-C/EBP $\beta-2$ ) were applied to knockdown the expression of $C / E B P \beta$. C/EBP $\beta$ was knocked down by over $60 \%$ by si-C/EBP $\beta-2$ as shown in Fig. 5a. Knocking down of $\mathrm{C} / \mathrm{EBP} \beta$ significantly promoted the cell viability (Fig. $5 \mathrm{~b}$ ) and increased the cell 
a
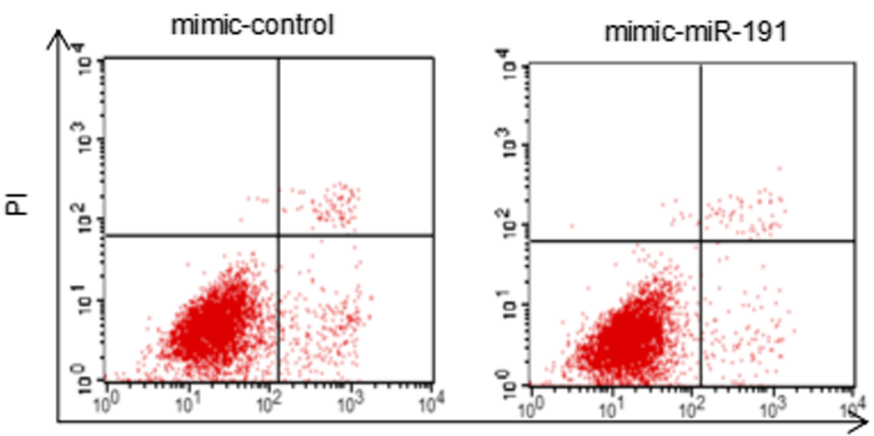

Annexin-V FITC

C
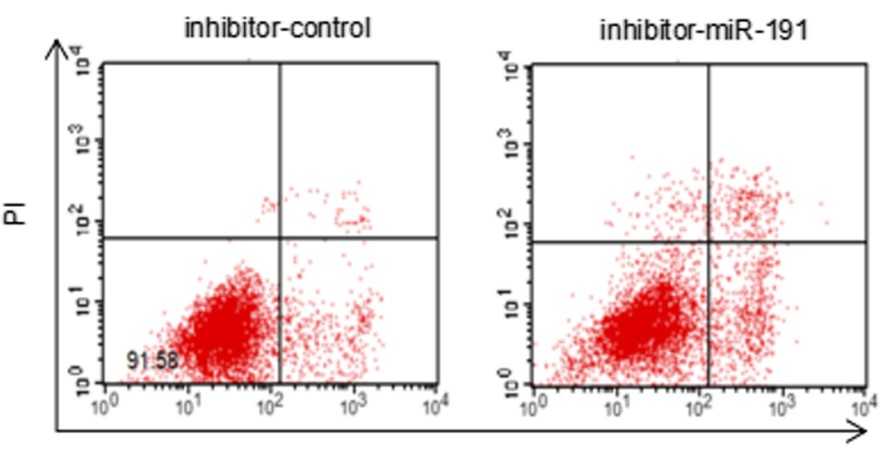

Annexin-V FITC

e
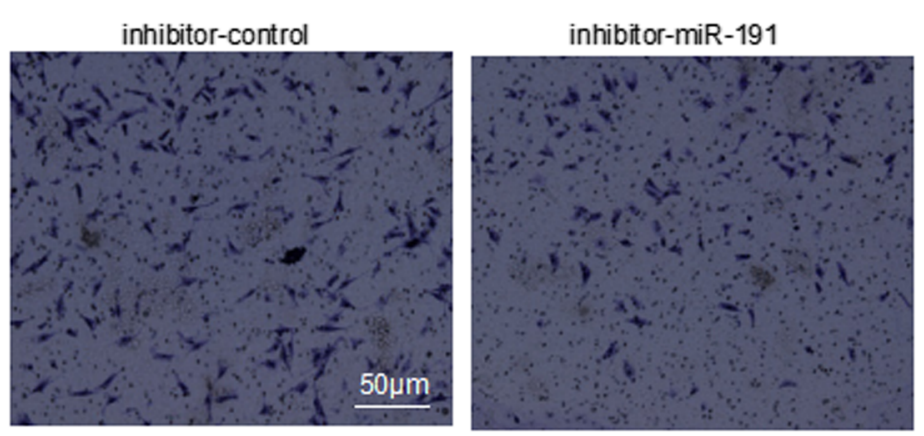

b

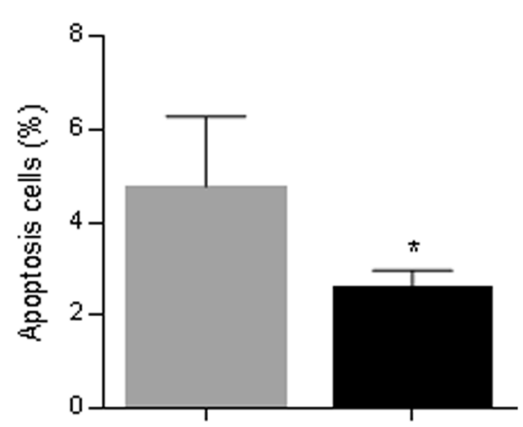

d

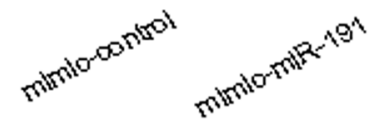

f
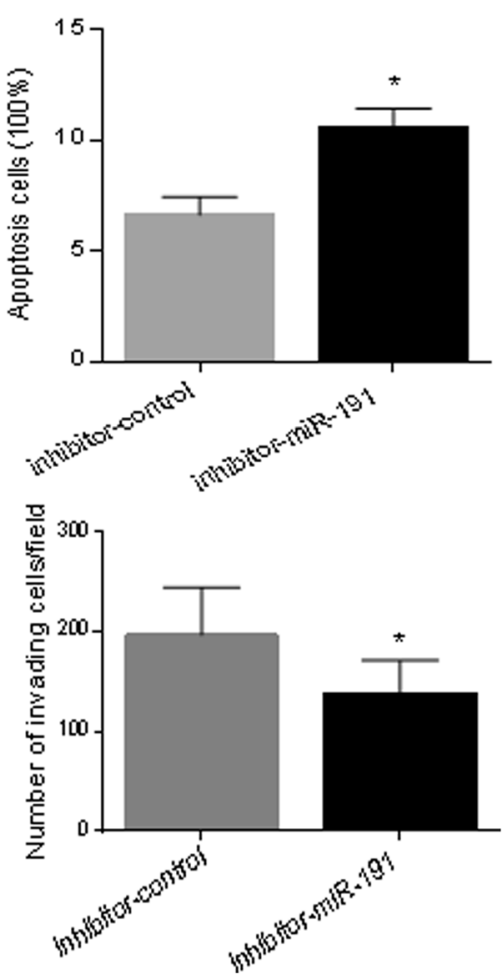

Fig. 3 miR-191 inhibited apoptosis in RA-FLS. a RA-FLS were starved with 1\% fetal serum for $24 \mathrm{~h}$ with or without overexpression of miR-191, followed by FACS analysis. b Bar graph showing the decreased apoptotic cell percentage after transfection with mimc-miR-191. c RA-FLS were starved with $1 \%$ fetal serum for $24 \mathrm{~h}$ with or without knockdown of miR-191, followed by FACS analysis. $\mathbf{d}$ Bar graph showing the increased apoptotic cell percentage after transfection with inhibitor-miR-191. e Transwell assays using RA-FLS cells after transfection with inhibitor-miR-191 for $24 \mathrm{~h}$ showed knockdown of miR-191 significantly suppressed the cell invasion of RA-FLS. $\mathbf{f}$ Quantitative analysis of e. All experiments were repeated three times. Data were presented as mean $\pm \operatorname{SEM}\left(n=3,{ }^{*} p<0.05\right.$ by $t$ test $)$

cycle $\mathrm{G}_{1} / \mathrm{S}$ transition in RA-FLS (Fig. $5 \mathrm{c}$ ). pEnter-C/ EBP $\beta$, a vector overexpressing C/EBP $\beta$ (Fig. $5 \mathrm{~d}$ ), was applied to perform rescue assay. As shown in Fig. 5e, mimic-miR-191 increased the cell proliferation of RA-FLS, which was reversed by reintroduction back of $\mathrm{C} / \mathrm{EBP} \beta$, indicating the regulation of cell proliferation by miR-191 in RA-FLS is dependent on the target gene C/ EBP $\beta$.
C/EBP $\beta$ involvement in the regulation of hypoxia-induced cell proliferation in RA-FLS

In order to further determine the role of $C / E B P \beta$ in RA-FLS, cells were cultured under hypoxia and normal condition, followed by gene expression assays. As shown in Fig. 6a and b, C/EBP $\beta$ was remarkably suppressed in expression by hypoxia at both mRNA and protein levels. Overexpression of C/EBP $\beta$ could partly rescue the 

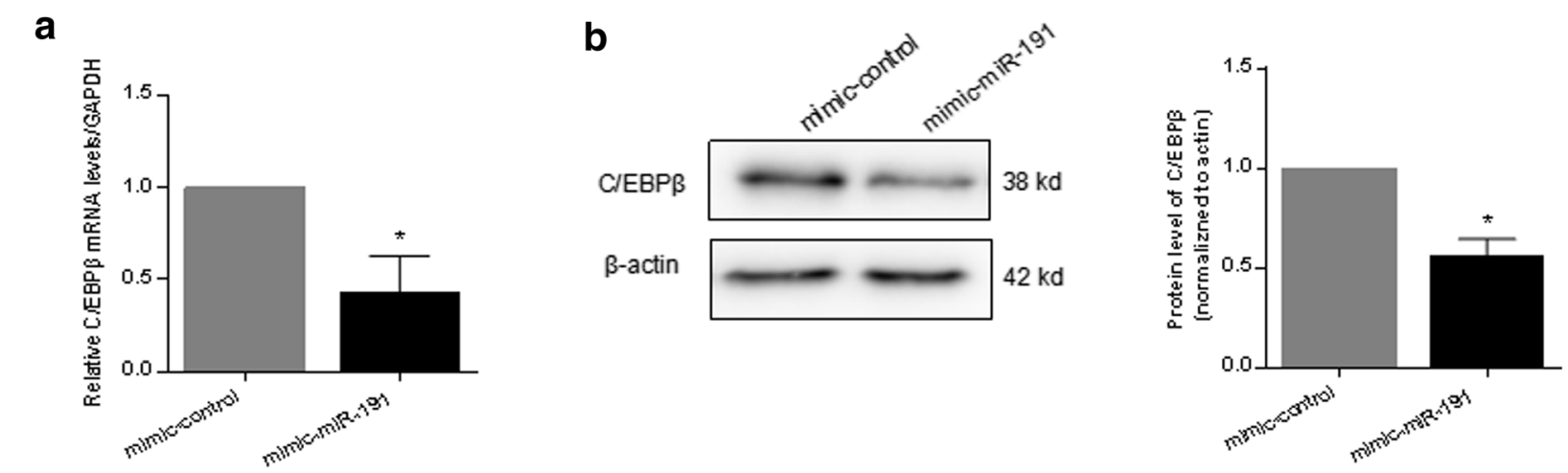

C

d
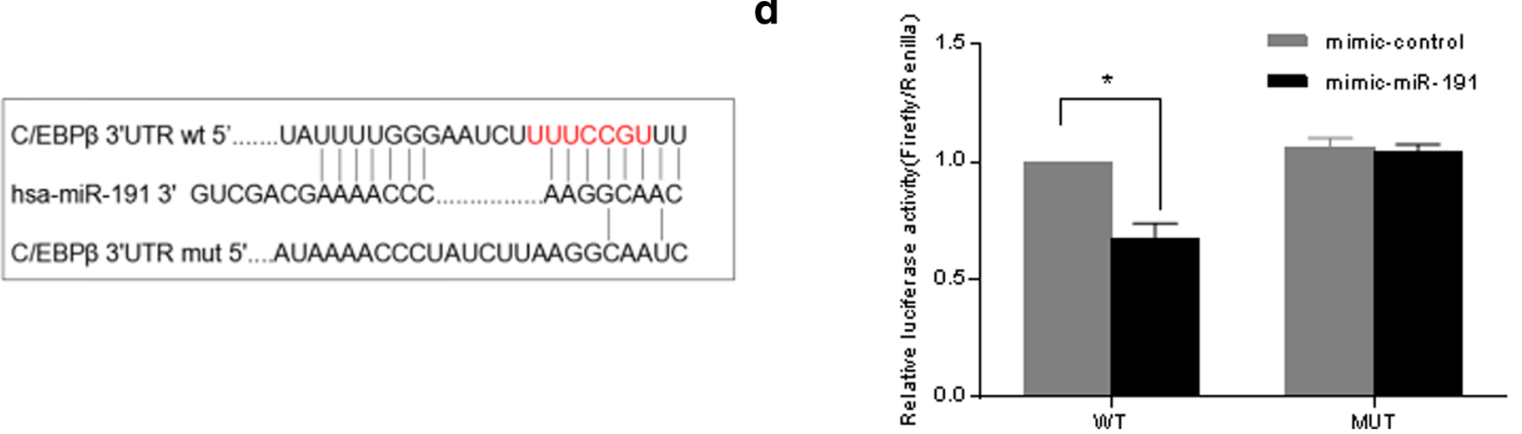

Fig. 4 miR-191 regulates C/EBP $\beta$ expression in RA-FLS. a quantitative RT-PCR analyses demonstrated the decrease of mRNA levels of C/EBP $\beta$ in RA-FLS after transfected with miR-191-mimic for $24 \mathrm{~h}$. $\mathbf{b}$ Western blot analyses demonstrated the decrease of C/EBP $\beta$ protein level in RA-FLS after transfected with miR-191-mimic for 48 h. c Sequences of the predicted miR-191 target sequences in the $3^{\prime}-U T R$ of C/EBP $\beta$ and its mutant containing altered nucleotides in the $3^{\prime}$-UTR. $\mathbf{d}$ Luciferase reporter assays demonstrated the inhibition of C/EBP $\beta$ 3'-UTR by direct interaction with miR-191. All experiments were repeated three times. Data were presented as mean \pm SEM $\left(n=3,{ }^{*} p<0.05\right.$ by $t$ test)

hypoxia-induced cell proliferation (Fig. 6c). The findings above suggested that miR-191-C/EBP $\beta$ signaling is required to mediate the hypoxia-induced cell proliferation in RA-FLS.

\section{Discussion}

Hypoxia has been well confirmed to be a common feature in most of the solid tumors [26]. It plays important roles in the development and progression of cancer [27]. Emerging evidence has demonstrated that it is inflammation and hypoxic microenvironment in the synovium that aggravates the cell proliferation and suppresses the cell apoptosis of synoviocytes in RA patients [28, 29].

In the current study, a miRNA screen analysis in the hypoxia-exposed RA-FLS was performed. We identified miR-297, miR-499b-3p, miR-770, miR-936, and miR-191 as the differently expressed miRNAs in RA-FLS under hypoxia condition. Interestingly, the upregulation of miR-191 was found to be specific in hypoxic RA-FLS, compared to hypoxic OA-FLS. The regulatory function of miR-191 to the cell proliferation and apoptosis of RA-FLS was further determined. We found miR-191-C/EBP $\beta$ mediated the upregulation of cell proliferation and inhibition of cell apoptosis in RA-FLS. Enforced overexpression of miR-191 was demonstrated to promote the proliferation and cell cycle and protect cells against apoptosis, suggesting miR-191 might be a potential target in RA treatment. In addition, upregulation of miR-191 was found in various types of cancer, autoimmune disease, and inflammatory disease [30-33]. In pancreatic cancer, miR-191 was found to suppress USP10, attenuated p53 stability, and thereby activate NF- $\mathrm{kB}$ signaling [34]. In consistence, proinflammatory cytokines including TNF- $\alpha$, interleukin(IL)- $1 \beta$, and IL-6, down-stream targets of NF-kB signaling, showed upregulation by miR-191 [34]. In view of the correlation between RA and inflammation, miR-191 is very likely to involve in the regulation of proinflammatory reaction in RA-FLS.

$\mathrm{C} / \mathrm{EBP} \beta$ has been shown to regulate cell proliferation, differentiation, and cell apoptosis in a variety of cell types $[35,36]$. We found that $C / E B P \beta$ is a functional target of miR-191 in RA-FLS. MiR-191 targeted C/EBP $\beta$ regulating cell cycle inhibitors p16, p15, and p57 in colorectal cancer and thus is involved in the cell cycle regulation [22]. However, the miR-191-C/EBP $\beta$ interaction and the regulatory function in RA-FLS have not been 


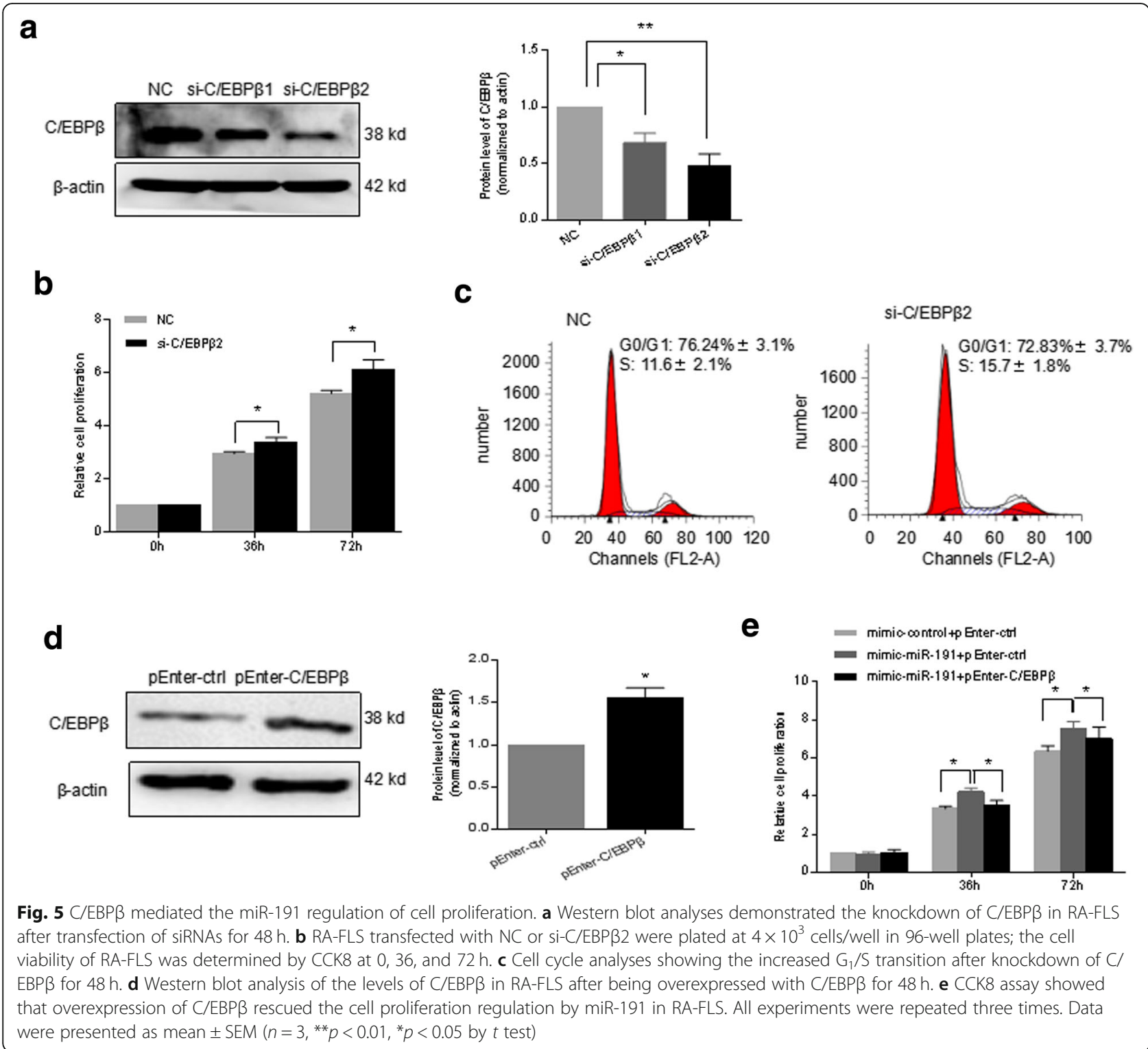

reported yet. Herein we showed that $C / E B P \beta$ was downregulated by miR-191 as a target gene in RA-FLS, and knocking down of C/EBP $\beta$ promoted RA-FLS proliferation significantly. Reintroduction $\mathrm{C} / \mathrm{EBP} \beta$ back to RA-FLS after hypoxia treatment could rescue the hypoxia-miR-191-induced phenotypes, suggesting the cell proliferation and apoptosis regulation by hypoxia-miR-191 are mediated, at least partly, by C/ EBP $\beta$. However, rescue experiments did not entirely recover normal phenotypes, and further studies are needed to detect the role of other targets or factors in the hypoxia-induced cell function. In addition to $C / E B P \beta$, ten-eleven translocation 1 (TET1) is also a predicted target of miR-191 in cholangiocarcinoma cells, which inhibits cell proliferation through p53 signaling pathway
[37]. Whether miR-191-TET1-p53 signaling pathway is also involved in the hypoxia-regulated cell function in RA-FLS is going to be experimentally determined by our ongoing study.

Except for proliferation induction, hypoxia has been demonstrated to promote VEGF secretion from RA-FLS and regulate angiogenesis in RA [38]. miR-191 was also found to be involved in HIF- $2 \alpha$-induced angiogenesis [39]. In breast cancer, loss of $\mathrm{C} / \mathrm{EBP} \beta$ was reported to promote breast cancer progression by shifting TGF $\beta$ response [40], which is an extremely strong stimulator of VEGF production by synovial fibroblasts $[41,42]$. Thus, these studies may be suggestive of the possible role of hypoxia-miR-191-C/EBP $\beta$ in regulating angiogenesis in RA, which needed to be further experimentally validated. 


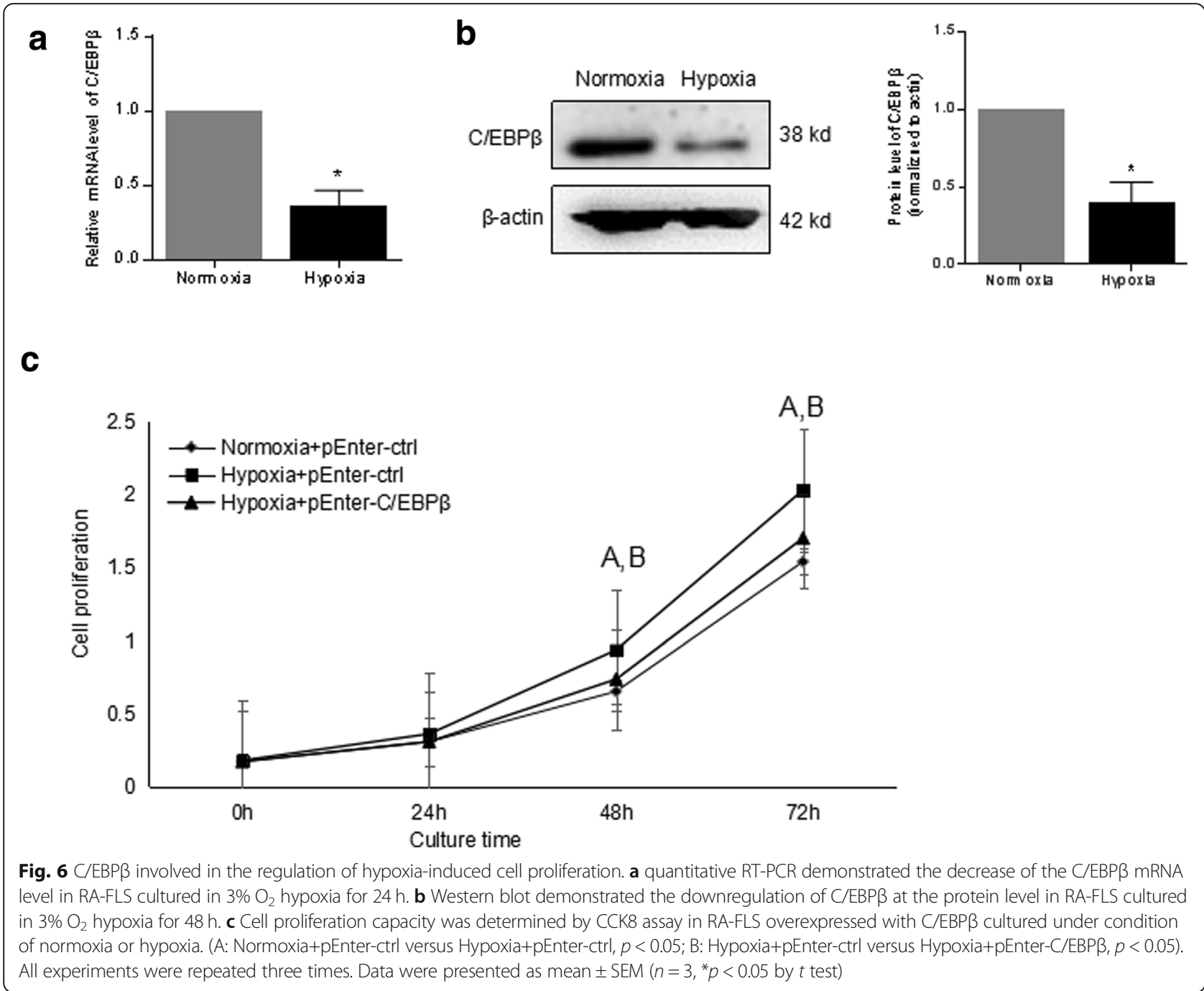

\section{Conclusions}

Taken together, our findings demonstrate that hypoxia-induced miR-191-C/EBP $\beta$ signaling is required to mediate the cell proliferation and apoptosis of RA-FLS. These findings provide new insights into the role of hypoxamiR in RA and suggested miR-191 is a promising therapeutic target for RA.

\section{Abbreviations}

3'-UTR: Three-prime untranslated region; ACR: American College of Rheumatology; C/EBP $\beta$ : CCAAT/enhancer binding protein $\beta$; CCK8: Cell Counting Kit-8; FBS: Fetal bovine serum; FLS: Fibroblast-like synoviocytes; G6PI: Glucose-6-phosphate isomerase; HE: Hematoxylin and eosin; HIFs: Hypoxia-inducible factors; hypoxamiR: Hypoxia-induced microRNAs; miRNA: MicroRNA; OA: Osteoarthritis; RA: Rheumatoid arthritis; RT-PCR: Real-time polymerase chain reaction; si-C/EBPB: C/EBP $\beta$ siRNA; si-NC: Si-negative control; TET1: Ten-eleven translocation 1; VEGF: Vascular endothelial growth factor

\section{Funding}

This study was supported by Project of Pudong Health Bureau of Shanghai (No. PW2013B-5), the Program for Key Discipline Construction Project of Pudong Health Bureau of Shanghai (No. PWZxk2017-09) and the National Natural Science Foundation of China (No. 81401303; No. 81671599).

\section{Availability of data and materials}

The datasets analyzed during the current study are available from the corresponding author on reasonable request.

\section{Authors' contributions}

All authors were involved in drafting the article or revising it critically for important intellectual content, and all authors approved the final version to be published. LYF had full access to all of the data in the study and takes responsibility for the integrity of the data and the accuracy of the data analysis. LYF was responsible for the study conception and design. SY, YL, $M Z$, and $Q T$ handled the acquisition of the data. SY, YL, and MZ handled the analysis and interpretation of data.

\section{Ethics approval and consent to participate}

This study was performed in accordance with the Declaration of Helsinki and was approved by the Ethics Committee of Shanghai East Hospital, Tongji University.

\section{Consent for publication}

Not applicable. 


\section{Competing interests}

The authors declare that they have no competing interests.

\section{Publisher's Note}

Springer Nature remains neutral with regard to jurisdictional claims in published maps and institutional affiliations.

\section{Author details}

'Department of Clinical Laboratory, Shanghai East Hospital, Tongji University School of Medicine, $150 \mathrm{Ji}$ Mo Road, Shanghai 200120, People's Republic of China. ${ }^{2}$ Research Center for Translational Medicine, Shanghai East Hospital, Tongji University School of Medicine, $150 \mathrm{Ji}$ Mo Road, Shanghai 200120 People's Republic of China.

\section{Received: 17 October 2018 Accepted: 6 March 2019}

Published online: 20 March 2019

\section{References}

1. Gibofsky A. Overview of epidemiology, pathophysiology, and diagnosis of rheumatoid arthritis. Am J Manag Care. 2012;18:S295-302.

2. Vital EM, Emery P. The development of targeted therapies in rheumatoid arthritis. J Autoimmun. 2008;31:219-27.

3. Filer A. The fibroblast as a therapeutic target in rheumatoid arthritis. Curr Opin Pharmacol. 2013;13:413-9.

4. Sivakumar B, Akhavani MA, Winlove CP, Taylor PC, Paleolog EM, Kang N. Synovial hypoxia as a cause of tendon rupture in rheumatoid arthritis. J HandSurg. 2008;33:49-58.

5. $\mathrm{Ng} \mathrm{CT}$, Biniecka M, Kennedy A, McCormick J, Fitzgerald O, Bresnihan B, Buggy D, Taylor CT, O'Sullivan J, Fearon U, Veale DJ. Synovial tissue hypoxia and inflammation in vivo. Ann Rheum Dis. 2010;69:1389-95.

6. Nonomura Y, Mizoguchi F, Suzuki A, Nanki T, Kato H, Miyasaka N, Kohsaka H. Hypoxia-induced abrogation of contact-dependent inhibition of rheumatoid arthritis synovial fibroblast proliferation. J Rheumatol. 2009;36:698-705.

7. MacDonald KV, Sanmartin C, Langlois K, Marshall DA. Symptom onset, diagnosis and management of osteoarthritis. Health Rep. 2014;25:10-7.

8. Glyn-Jones S, Palmer AJ, Agricola R, Price AJ, Vincent TL, Weinans H, Carr AJ. Osteoarthritis. Lancet. 2015;386:376-87.

9. Lund-Olesen K. Oxygen tension in synovial fluids. Arthritis Rheum. 1970;13: 769-76.

10. Biniecka M, Connolly M, Gao W, Ng CT, Balogh E, Gogarty M, Santos L, Murphy E, Brayden D, Veale DJ, Fearon U. Redox-mediated angiogenesis in the hypoxic joint of inflammatory arthritis. Arthritis Rheumatol. 2014;66: 3300-10.

11. Akhavani MA, Madden L, Buysschaert I, Sivakumar B, Kang N, Paleolog EM. Hypoxia upregulates angiogenesis and synovial cell migration in rheumatoid arthritis. Arthritis Res Ther. 2009;11:R64.

12. Konisti S, Kiriakidis S, Paleolog EM. Hypoxia--a key regulator of angiogenesis and inflammation in rheumatoid arthritis. Nat Rev Rheumatol. 2012:8:153-62.

13. Lu Y, Yu SS, Zong M, Fan SS, Lu TB, Gong RH, Sun LS, Fan LY. Glucose-6 phosphate isomerase (G6PI) mediates hypoxia-induced angiogenesis in rheumatoid arthritis. Sci Rep. 2017;7:40274.

14. Greco S, Gaetano C, Martelli F. HypoxamiR regulation and function in ischemic cardiovascular disease. Antioxid Redox Signal. 2014;21:1202-19.

15. Gee HE, Camps C, Buffa FM, Patiar S, Winter SC, Betts G, Homer J, Corbridge R, Cox G, West CM, Ragoussis J, Harris AL. hsa-mir-210 is a marker of tumor hypoxia and a prognostic factor in head and neck cancer. Cancer. 2010;116: 2148-58.

16. Camps C, Saini HK, Mole DR, Choudhry H, Reczko M, Guerra-Assunção JA, Tian YM, Buffa FM, Harris AL, Hatzigeorgiou AG, Enright AJ, Ragoussis J. Integrated analysis of microRNA and mRNA expression and association with HIF binding reveals the complexity of microRNA expression regulation under hypoxia. Mol Cancer. 2014;13:28.

17. Bartel DP. MicroRNAs: genomics, biogenesis, mechanism, and function. Cell. 2004:116:281-97.

18. Nakasa T, Miyaki S, Okubo A. Expression of microRNA-146 in rheumatoid arthritis synovial tissue. Arthritis Rheum. 2008;58:1284-92.

19. Migita K, Iwanaga N, Izumi Y, Kawahara C, Kumagai K, Nakamura T, Koga T, Kawakami A. TNF-a-induced miR-155 regulates IL-6 signaling in rheumatoid synovial fibrolasts. BMC Res Notes. 2017;10:403.
20. Sharma S, Nagpal N, Ghosh PC, Kulshreshtha R. P53-miR-191-SOX4 regulatory loop affects apoptosis in breast cancer. RNA. 2017;23:1237-46.

21. Elyakim E, Sitbon E, Faerman A, Tabak S, Montia E, Belanis L, Dov A, Marcusson EG, Bennett CF, Chajut A, Cohen D, Yerushalmi N. hsa-miR-191 is a candidate oncogene target for hepatocellular carcinoma therapy. Cancer Res. 2010;70:8077-87.

22. Zhang XF, Li KK, Gao L, Li SZ, Chen K, Zhang JB, Wang D, Tu RF, Zhang JX, Tao KX, Wang G, Zhang XD. miR-191 promotes tumorigenesis of human colorectal cancer through targeting C/EBP $\beta$. Oncotarget. 2015;6:4144-58.

23. Pal R, Janz M, Galson DL, Gries M, Li S, Jöhrens K, Anagnostopoulos I, Dörken B, Mapara MY, Borghesi L, Kardava L, Roodman GD, Milcarek C, Lentzsch S. C/EBPbeta regulates transcription factors critical for proliferation and survival of multiple myeloma cells. Blood. 2009;114:3890-8.

24. Tsukada J, Yoshida Y, Kominato Y, Auron PE. The CCAAT/enhancer (C/EBP) family of basic-leucine zipper (bZIP) transcription factors is a multifaceted highly-regulated system for gene regulation. Cytokine. 2011:54:6-19.

25. Ramji DPD, Foka P. CCAAT/enhancer-binding proteins: structure, function and regulation. Biochem J. 2002;365:561-75.

26. Laitala A, Erler JT. Hypoxic signalling in tumour stroma. Front Oncol. 2018;8:189.

27. Yang ZF, Poon RT, To J, Ho DW, Fan ST. The potential role of hypoxia inducible factor 1 alpha in tumor progression after hypoxia and chemotherapy in hepatocellular carcinoma. Cancer Res. 2004:64:5496-503.

28. Lee SY, Kwok SK, Son HJ, Ryu JG, Kim EK, Oh HJ, Cho ML, Ju JH, Park SH, Kim HY. IL-17-mediated Bcl-2 expression regulates survival of fibroblast-like synoviocytes in rheumatoid arthritis through STAT3 activation. Arthritis Res Ther. 2013;15:R31.

29. Ryu JH, Chae CS, Kwak JS, Oh H, Shin Y, Huh YH, Lee CG, Park YW, Chun CH, Kim YM, Im SH, Chun JS. Hypoxia-inducible factor-2a is an essential catabolic regulator of inflammatory rheumatoid arthritis. PLoS Biol. 2014;12: e1001881.

30. Peng WZ, Ma R, Wang F, Yu J, Liu ZB. Role of miR-191/425 cluster in tumorigenesis and diagnosis of gastric cancer. Int J Mol Sci. 2014;15:4031-48.

31. Wang T, Ji F, Dai Z, Xie Y, Yuan D. Increased expression of microRNA-191 as a potential serum biomarker for diagnosis and prognosis in human osteosarcoma. Cancer Biomark. 2015:15:543-50.

32. Vistbakka J, Sumelahti ML, Lehtimäki T, Elovaara I, Hagman S. Evaluation of serum miR-191-5p, miR-24-3p, miR-128-3p, and miR-376c-3 inmultiple sclerosis patients. Acta Neurol Scand. 2018;138:130-6.

33. Paraskevi A, Theodoropoulos G, Papaconstantinou I, Mantzaris G, Nikiteas N, Gazouli M. Circulating MicroRNA in inflammatory bowel disease. J Crohns Colitis. 2012;6:900-4

34. Liu H, Xu XF, Zhao Y, Tang MC, Zhou YQ, Lu J, Gao FH. MicroRNA-191 promotes pancreatic cancer progression by targeting USP10. Tumour Biol. 2014:35:12157-63.

35. Grimm SL, Rosen JM. The role of C/EBPbeta in mammary gland development and breast cancer. J Mammary Gland Biol. 2003:8:191-204.

36. Lekstrom-Himes J, Xanthopoulos KG. Biological role of the CCAAT/ enhancer-binding protein family of transcription factors. J Biol Chem. 1998; 273:28545-8.

37. Li H, Zhou ZQ, Yang ZR, Tong DN, Guan J, Shi BJ, Nie J, Ding XT, Li B, Zhou GW, Zhang ZY. MicroRNA-191 acts as a tumor promoter by modulating the TET1-p53 pathway in intrahepatic cholangiocarcinoma. Hepatology. 2017;66: 136-51.

38. Kennedy A, Ng CT, Biniecka M, Saber T, Taylor C, O'Sullivan J, Veale DJ, Fearon U. Angiogenesis and blood vessel stability in inflammatory arthritis. Arthritis Rheum. 2010;62:711-21.

39. Xu W, Luo F, Sun B, Ye H, Li J, Shi L, Liu Y, Lu X, Wang B, Wang Q, Liu Q, Zhang A. HIF-2a, acting via miR-191, is involved in angiogenesis and metastasis of arsenite-transformed HBE cells. Toxicol Res (Camb). 2015:5:66-78.

40. Johansson J, Berg T, Kurzejamska E, Pang MF, Tabor V, Jansson M, Roswall P, Pietras K, Sund M, Religa P, Fuxe J. MiR-155-mediated loss of C/EBP $\beta$ shifts the TGF- $\beta$ response from growth inhibition to epithelial-mesenchymal transition, invasion and metastasis in breast cancer. Oncogene. 2013;32:5614-24.

41. Pertovaara L, Kaipainen A, Mustonen T, Orpana A, Ferrara N, Saksela O, Alitalo K. Vascular endothelial growth factor is induced in response to transforming growth factor- $\beta$ in fibroblastic and epithelial cells. J Biol Chem. 1994;269:6271-4.

42. Berse B, Hunt JA, Diegel RJ, Morganelli P, Yeo K, Brown F, Fava RA. Hypoxia augments cytokine (transforming growth factor-beta (TGF-beta) and IL-1)induced vascular endothelial growth factor secretion by human synovial fibroblasts. Clin Exp Immunol. 1999;115:176-82. 